\title{
Morteros hidráulicos de cal y chamota
}

\author{
Aired-lime and chamotte hydraulic mortars \\ M. GONZÁLEZ CORTINA" ${ }^{\prime \prime}$, I. de VIILANLEVA DOMÍNGUEZ ${ }^{(-\infty)}$ \\ ${ }^{(")}$ E.U.A.T. de Madrid, ${ }^{\text {"* }}$ E.T.S.A. de Madrid
}

Fecha de recepción: 2-VII-()I

Fecha de aceptación: 9-X-01

ESPANA

\section{RESUMEN}

El objetivo de éste trabajo es el conseguir morteros hidráulicos, a partir de la cal aérea, con adición de chamota o polvo de arcilla cocida, obtenida de los residuosdesechos de la industria cerámica. De este modo se pretende infundir propiedades hidráulicas a la cal y obtener morteros hidráulicos, con una mejora sustancial de sus propiedades mecánicas.

Para ello, se ha experimentado con diversos tipos de chamotas, obtenidas a partir de arcillas cocidas a diferentes temperaturas, y con diversas granulometrias, y se han realizado morteros con distintas dosificaciones, variando las proporciones de cal, arena, chamota y agua.

En todos los casos se ha preparado una doble serie de probetas, para conservarlas al aire o sumergidas en agua, sobre las que se han determinado la dureza Shore $C$ y la resistencia mecánica a flexión y compresión. Posteriormente se han efectuado análisis mediante difracción por rayos $X$, para determinar cualitativamente la composición de la estructura cristalina, así como análisis microestructurales, por medio de estereomicroscopía y por microscopía electrónica de barrido, con microanálisis de ravos $X$.

Como conclusión del trabajo, se han formulado varios tipos de morteros con distintas dosificaciones, que, por sus características, son válidos como morteros de agarre en fábricas de albañileria, tanto para las obras de restauración como para las de obra nueva.

\section{SUMMARY}

The aim of this research project has been to obtain airedlime based hydraulic mortars with the addition of chamotte or burnt clay powder obtained from the ceramic industry vaste. By doing this, hydraulic properties have been included into lime and hydraulic mortars with a great improvement in mechanical properties.

In order to achieve this, different types of chamotte obtained from clay burnt at different temperatures have been tested. changing, at the same time, the proportions of lime, sand, chamotte and water:

All the tests have been performed preparing a double set of test pieces to be kept at room temperature or to be immersed in vater, determining the Shore C hardness and the mechanical compressive and tensile strengths. Further on, $X$ ray diffraction analysis have been developed to determine the qualitative composition of the crystalline structure, as well as micro structural analysis, using stereomicroscope and electric microscope scanning, with X-ray microanalysis have been used.

As a conclusion, several types of mortars have been created with different proportions, which can be used, due to its characteristics, as keying mortars in brickwork, for restoration works as well as for new constructions.

\section{INTRODUCCIÓN}

Desde la antigüedad romana, se vienen utilizando materiales puzolanicos, naturales o artificiales, como adiciones para favorecer la hidraulicidad de conglomerantes no hidráulicos. Uno de estos materiales ha sido el polvo de arcilla cocida o chamota que se añadían a los morteros de cal.

\section{INTRODUCTION}

From Roman times, natural or artificial pusolanic materials have been used, as well as additives to encourage hydraulicity of non-hydraulic agglomerates. One of the materials has been burnt clay dust or (hamotte that is added to the lime mortars. 
Hay numerosos ejemplos de la utilización de estos morteros, empleando distintas granulometrías, como la bóveda de cañón que cubre la piscina principal de los baños de Bath en Inglaterra, del S. I, d. C., la Iglesia de San Lorenzo de Milán del S. V d. C., los Santos Sergio y Baco en Estambul del S. VI d. C., etc. (1).

Los morteros de cal utilizados tradicionalmente cayeron en desuso a partir de la Revolución Industrial y fueron sustituidos indiscriminadamente por morteros de cemento. Sin embargo las características de los morteros de cal como la mejora de la plasticidad y trabajabilidad, incremento de la retención de agua, curado autógeno, etc., les hacen insustituibles para determinados tipos de trabajo, entre ellos los de restauración (2). Es por ello que a partir de la década de los ochenta se han ido recuperando, aunque de forma remisa, para los trabajos antes mencionados.

Este trabajo se basa en la adición de polvo de ladrillo procedente de desechos de la industria cerámica, tal y como se ha expuesto anteriormente, de tal manera que se efectúa un reciclaje de esos productos de desecho para añadirlos a la cal aérea, y conferirle, de esta manera, propiedades hidráulicas a la cal.

El fundamento científico de esta adición, que de forma empírica se venía utilizando tradicionalmente, se basa en la denominada actividad puzolánica. Se consideran generalmente puzolanas los materiales que carentes de propiedades cementicias y de actividad hidráulica por si solos, contienen constituyentes que se combinan con la cal a temperaturas ordinarias y en presencia del agua, dando lugar a compuestos permanentemente insolubles y estables, que se comportan como conglomerantes hidráulicos.

Hay diversos tipos de ensayos para comprobar la hidraulicidad de los conglomerantes, pastas y morteros, pudiendo distinguirse básicamente entre ensayos que estudian únicamente las puzolanas(solubilidad, composición química, etc.), ensayos de cal y puzolanas y ensayos de cemento y puzolana.

En este trabajo se ha utilizado el denominado ensayo físico de hidraulicidad que objetiviza de forma cómoda y fiable la hidraulicidad, basándose en los resultados de ensayos mecánicos entre amasadas conservadas al aire en condiciones normales de laboratorio y amasadas conservadas sumergidas en agua.

Para la realización de este ensayo se realizaron diversas amasadas, con distintos conglomerantes y distintas dosificaciones.

De todas ellas se realizaron amasadas para romper a edades de 2,7 y 28 días, duplicando el número de.
There are many examples of these mortars, using granulometries, such as the vaulted dome in the Roman baths in Bath (England) of the I"t c. after $C$., the church of San Loren:o in Milan from the 5thc. after C., the Saints Serge and Bacus in Istambul from the $6^{\text {th }}$ c. after C., etc. (1).

Cement mortars substituted the traditionally used lime mortars at the time of the Industrial Revolution. Nevertheless, lime mortar characteristics, such as plasticity and workability, increase in water retention, autogenous setting etc., make these mortars of great value for certain types of works, especially in restauration (2). Because of this, lime mortars have slowly been recuperated since the 80's.

This research article deals with the addition of brick powder from ceramic industry waste. The recycling of those waste products to be added to the aired-lime mortars incorporates hydraulic properties to lime.

The scientific principle of this addition, which has been used experimentally a long time ago, is based on the puzzolanic activity. Puzzolanas are those which, although lacking lime properties and hydraulic activity on their ow'n, they do contain constituents which are combined with lime at ordinary temperature and with water presence, so as to form permanently stable and insoluble compounds. These compounds behave as hydraulic conglomerates.

There are several tests to study the hydraulicity of the conglomerates, pastes and morlars. Three types can be distinguished: tests that studies only puzzolanas (solubility, chemical composition, etc.), lime and puzzolana tests and cement and puz:olana tests.

For this research, the physical test for hydraulicity, which quantifies easy and reliably the hydraulicity, has been used,. It bases its results in the mechannical tests performed in mixings conserved at room temperature and mixings immersed in water.

In order to perform this tests, several mixings were done using different types of conglomerates and different proportions.

Tivo sets of test mixings were produced to be analysed at 2.7 and 28 days, one of the sets to be kept at room 
amasadas para conservarlas tanto al aire, en condiciones normales de humedad y temperatura, correspondientes a las condiciones de laboratorio, las más asemejables a las de obra, como sumergidas en agua desde cl momento de su fabricación.

El objeto de sumergirlas en agua, condiciones por otra parte no habituales en morteros de agarre en obra, se eligieron por considerarse la constatación última de la hidraulicidad de los distintos materiales.

De este ensayo se obtuvieron una serie de conclusiones, válidas para el fin que se pretendía, siendo las que más disparidad presentaban, aquéllas que contenían cal aérea en su composición (3).

\section{PARTE EXPERIMENTAL}

El objetivo de cste estudio es comprobar qué granulometrías y temperaturas de cocción son las adecuadas para la utilización de la chamota como adición de las cales aéreas para la obtención de cales hidráulicas para su uso en morteros de agarre para obra nueva y restauración, para, posteriormente, definir las dosificaciones más idóneas.

Para ello se han realizado distintas amasadas utilizando diversas granulometrías de la chamota, refiriéndola al módulo granulométrico, forma habitual de clasificar los áridos por su tamaño, expresado como el sumatorio de los tantos por ciento retenidos y acumulados de los diez tamices de la norma UNE 7.050 dividido por cien. De esta manera se ha trabajado con distintos módulos granulométricos de la chamota que van desde 3,94 , la mas gruesa, a 0,32 la más fina. Para la obtención de estas granulometrías se ha procedido al triturado previo del ladrillo cocido y posterior tamizado.

Las temperaturas de cocción utilizadas han sido las de $1.300{ }^{\circ} \mathrm{C}$ y $950-1.000{ }^{\circ} \mathrm{C}$, temperaturas habituales en la fabricación de los productos cerámicos,

correspondientes las primeras a los tipos gresificados y las segundas a las temperaturas utilizadas en la fabricación de ladrillos, tejas, etc.

No se han utilizado temperaturas distintas a las indicadas, estudiadas por otros autores (4) (5), porque lo que se pretende es el aprovechamiento de materiales residuales para favorecer los efectos sinérgicos de materiales compuestos y aditivados con adiciones de bajo costo.

De las distintas amasadas realizadas, se ha medido en todas ellas las durezas Shore $\mathrm{C}$ a distintas edades así como ensayos para la obtención de resistencias mecánicas a flexión y compresión, comprobando la evolución a lo largo del tiempo y comparándolas entre las probetas normales y sumergidas. temperature (normal humidity conditions) similar to work site conditions and the other set was kept immersed in water:

The aim of study for the mixings immersed in water, not common conditions for keying mortars- lias to analyse the most extreme case of hydraulicity in the different materials.

From the tests performed, the results, which showed greater disparity, were fiom those mixings, which contained aired lime in their composition (3).

\section{EXPERIMENTAL PROCESS}

The aim of this study is to explore the appropriate grading and the kilning temperature of chamotte used as an additive in aired limes in order to obtain hydraulic limes used as keying mortars in restauration as well as in new construction works. Later, the most suitable proportions will be defined.

Several mixings have been made using different chamotte gradings, with reference to the granulemetric module, a common way of classifying aggregates by their size. This is expressed by the summitry of the retained and accumulated percentage of the ten sieves of the UNE 7.050 standard divided by a hundred. Several chamotte granulemetric modules have been used ranging from 3.94 for the greatest to 0.32 for the smallest. To obtain these grain sizes burnt brick crushing has been performed first and later sieving has been done.

The kilning temperatures used have been 1.300 " $\mathrm{C}$ and 950-1.000 "C, common temperatures in ceramic products. The first one corresponds to earthenware types and the second ones to the temperatures used in the production of bricks, tiles, etc.

No other temperatures have been used, even though studied by other authors (4) (5), because the aim is to use waste materials to improve the synergic effects of compound materials and to additivate them with low cost materials.

From the different mixings done, the Shore C hardness has been studied at different timings, as well as tests for the compression and flexion mechanical strengths, testing the evolution through time and comparing them among the normal test pieces and the ones immersed in water. 
Para lo cual se han confeccionado probetas normalizadas de $40 \times 40 \times 160 \mathrm{~mm}$ según la norma UNE-EN 459-2, modificando las proporciones de los componentes.

Esquemáticamente se ha seguido el siguiente proceso:

- Dosificación de los componentes en peso.

- Amasado con amasadora planetaria.

- Enmoldado y compactado en compactadora automática.

- Conservación tanto al aire como sumergida en agua desde el momento de la realización de las probetas.

Se ha realizado análisis microestructural y DRX utilizando las siguientes técnicas instrumentales:

Microscopia estereoscópica o Estereomicroscopía: con un equipo Nikon SMZ-27 con sistema fotográfico Nikon Microflex AFX-DX y cámara fotográfica Nikon FX-35 DX.

Microscopía electrónica de barrido (SEM) combinada con Microanálisis por energías dispersivas de rayos $\mathrm{X}$ (EDX): realizadas con un microscopio JEOL 5400 y un microanálisis de la marca Oxford, modelo Link, sistema ISIS.

Los análisis efectuados han sido los siguientes:

Estereomicroscopía: aspecto general y detalle de la microestructura de las distintas muestras.

Microscopía electrónica de barrido con microanálisis de rayos X (SEM-EDX): aspecto general de la muestra con análisis de la composición promedio, aspecto de detalle de la microestructura de la muestra y puntos singulares, si existen.

Han sido analizadas las mismas probetas que en la difracción por RX, a 7, 28 y 120 días y tanto conservadas al aire como sumergidas en agua:

Para la determinación cualitativa de la composición de la estructura cristalina se han realizado ensayos de difracción por rayos $\mathrm{X}$, empleando un equipo automático de DRX marca Philips modelo 1730.

\section{RESULTADOS Y DISCUSIÓN}

3.1. Resultados obtenidos con chamotas cocidas a $1.3000^{\circ} \mathrm{C}$ y granulometría gruesa, módulo granulométrico 3,94 .
In order to do this, several test pieces have been produced of 40 $\times 40 \times 160 \mathrm{~mm}$ following standard UNE-EN 459-2, changing the components proportions.

The process followed has been the following:

- components proportion varying in weight.

- mixing with planetary clay mixer

- molding and compacting in automatic compactor

- conservation from the moment the test pieces were produced immersed in water or air kept.

Micro structural analysis and DRX analysis have been made using the following instrumental techniques:

Stereoscopic microscopy or stereomicroscopy: using a Nikon SMZ-27 with photographic system Nikon Microflex AFX-DX and camera Nikon FX-35 DX.

Electronic microscopy de BARRIDO (SEM) combined with Microanalysis by disperse $X$-ray energies (EDX) produced with a JEOL 5400 microscope and a microanalysis Oxford, Link model, ISIS system.

The analysis performed have been:

Stereomicroscopy: general aspect and detail of the microstructure of the different test pieces.

Electronic microscopy sweep with $X$ ray microanalysis (SEM-EDX): general aspect of the test pieces studying the average composition, detailed aspect of the test piece microstructure and singular points if there were any.

The same test pieces as in the Rx diffraction have been analyzed, at 7,28 and 120 days and both conserved in the air or in water.

For the qualitative determination of the crystaline structure composition several diffraction by X-ray tests have been performed, using a DRX automatic equipment, by Phillips, 1730 model.

\section{RESULTS AND DISCUSSION}

3.I. Results obtained with chamotte burned at $2.300^{\circ} \mathrm{C}$ and course granulometry, granulemetric module 3,94. 


\subsubsection{Conservadas al aire}

Como puede comprobarse en la Tabla I, los valores obtenidos en durezas superficiales Shore C son válidos, incluso para edades tempranas. Sin embargo las resistencias a flexión y compresión son muy bajas.

\subsubsection{Conservadas sumergidas en agua}

Las probetas sumergidas en agua, no endurecieron prácticamente, no pudiéndose, por lo tanto, aportar resultados.

3.2. Resultados obtenidos con chamotas cocidas a $1.000^{\circ} \mathrm{C}$ y granulometría gruesa, módulo granulométrico 3,94.

\subsubsection{Conservadas al aire}

Como puede comprobarse en la Tabla II, los valores obtenidos en durezas superficiales Shore C son válidos, incluso para edades tempranas. Sin embargo las resistencias a flexión y compresión son muy bajas.

\subsubsection{Conservadas sumergidas en agua}

Las probetas sumergidas en agua, no endurecieron prácticamente, no pudiéndose, por lo tanto, aportar resultados.

3.3. Resultados obtenidos con chamotas cocidas a $1.000^{\circ} \mathrm{C}$ y granulometría fina, módulo granulométrico 0,32 .

\subsubsection{Conservadas al aire}

Como puede comprobarse en la Tabla III y gráficos 1, 2 y 3 , las durezas superficiales van aumentando con el tiempo, si bien no de una forma proporcional. En cualquier caso, todas las amasadas experimentan un incremento de dureza superficial.

Para resistencias a flexión los mejores resultados se obtienen con una proporción de chamota de 1,5 en relación al peso de la cal, así como con una proporción 2 en arena. El contenido de agua influye también negativamente y a mayor cantidad de agua se obtienen menores resistencias a flexión.

Para resistencias a compresión volvemos a obtener los mejores resultados con chamota/cal $=1,5$, también con la menor cantidad de agua. Usando la misma dosificación y añadiendo arena con una relación arena/cal $=2$, las resistencias obtenidas son también satisfactorias.

Como puede comprobarse en la Tabla IV y gráficos 4,5 y 6 , las durezas superficiales van aumentando con el tiempo

\subsubsection{Air kept pest pieces:}

As can be seen on Table I, the obtained lalues in superficial hardness Shore $C$ are valid, even at an early age. Nevertheless, the flexion and compressive strengths are very low:

\subsubsection{Water immersed test pieces:}

The water immersed test pieces, did barely harden, therefore, we could not obtain results.

3.2. Results obtained with chamotte burned at $1.000^{\circ} \mathrm{C}$ and coarse granulometry, granulmetric module 3,94.

\subsubsection{Air kept test pieces}

As can be seen on Table II, the values obtain for Shore $C$ superficial hardness are valid, even at an early age. Nevertheless, the flexion and compressive strengths are very low:

\subsubsection{Water immersed test pieces}

The water immersed test pieces, did barely harden, therefore, we could not obtain results.

3.3. Results obtained with chamotte burned at $1.000^{\circ} \mathrm{C}$ and fine granulometry, granulemetric module 0,32.

\subsubsection{Air kept test pieces:}

As can be seen on Table III and graphs 1, 2, and 3, the superficial hardness increases with time, although not proportionally. In every mixing there is an increase in superficial hardness.

For flexion strength, the best results were obtained with a proportion of chamotte of 1.5 in relation to lime weight, as well as with a proportion 2 in sand. The water content affect negatively, and the greater the water content, the lower the flexion strength obtained.

For compressive strength, better results are obtained with chamotte /lime $=1.5$, also with the smallest amount of water. Using the same proportion and adding sand in a sand/lime ratio $=2$, the strengths obtained are also satisfactory.

As can be seen on Table III and graphs 4, 5, and 6, the superficial hardness increases irregularly with time, 
TABLA I/TABLE I

Granulometría gruesa, $1.300^{\circ} \mathrm{C}$. Conservadas al aire

(Course granulometry, $1.300^{\circ} \mathrm{C}$. Air kept test pieces)

\begin{tabular}{|c|c|c|c|c|c|c|c|c|c|}
\hline \multirow[t]{3}{*}{$\begin{array}{l}\text { A m as ad as } \\
\text { Mixings }\end{array}$} & \multicolumn{5}{|c|}{$\begin{array}{l}\text { Durezas superficiales Shore C } \\
\text { shore C superfitial hardness }\end{array}$} & \multicolumn{2}{|c|}{$\begin{array}{l}\text { Resistencia a flexión } \\
\text { en M pa } \\
\text { Flexion strength Mpa }\end{array}$} & \multicolumn{2}{|c|}{$\begin{array}{c}\text { Resistencia a } \\
\text { compresión en M pa } \\
\text { Compressive strength } \\
M p a\end{array}$} \\
\hline & \multicolumn{5}{|c|}{ Días/Days } & \multicolumn{2}{|c|}{ Días/Days } & \multicolumn{2}{|c|}{ Días/Days } \\
\hline & 7 & 14 & 21 & 28 & 90 & 28 & 90 & 28 & 90 \\
\hline $1: 1: 3$ & 31,00 & 72,00 & .00 & 86,00 & 90,00 & 0,45 & 0 , & 0 , & 0 . \\
\hline $1: 1: 2: 1,1$ & 22,00 & 60,00 & 72,00 & 83,00 & 84,00 & 0 & 0,55 & 0, & 0 , \\
\hline $1: 1,5: 3: 1,3$ & 27,00 & 66,00 & 78,00 & 84,00 & 84,00 & 0,50 & - & 0,34 & 0,50 \\
\hline $1: 1,5: 2: 1,1$ & 46,00 & 79,00 & 82,00 & 86,00 & 87,00 & 0,55 & 0,70 & 0,38 & 0,53 \\
\hline & 38,00 & 74,00 & 83,00 & 87,00 & 89,00 & & 0,70 & 0,35 & 0,65 \\
\hline $1: 0,5: 2: 1$ & 21,00 & 54,00 & 70,00 & 78,00 & 86,00 & 0,47 & 0,55 & 0,37 & 0,50 \\
\hline
\end{tabular}

TABLA II/TABLE II

Granulometría gruesa, $1.000^{\circ} \mathrm{C}$. Conservadas al aire

(Course granulometry, $1.000^{\circ} \mathrm{C}$. Air kept test pieces)

\begin{tabular}{|c|c|c|c|c|c|c|c|c|c|}
\hline \multirow[t]{3}{*}{$\begin{array}{c}\text { A m a a d as } \\
\text { Mixings }\end{array}$} & \multicolumn{5}{|c|}{$\begin{array}{c}\text { Durezas superficiales Shore C } \\
\text { Shore C superfitial hardness }\end{array}$} & \multicolumn{2}{|c|}{$\begin{array}{c}\text { Resistencia a flexión } \\
\text { en Mpa/Flexion } \\
\text { strength Mpa }\end{array}$} & \multicolumn{2}{|c|}{$\begin{array}{c}\text { Resistencia a } \\
\text { compresión en } \mathrm{M} \text { pa } \\
\text { Compressive strength } \\
\text { Mpa }\end{array}$} \\
\hline & \multicolumn{5}{|c|}{ Días/Days } & \multicolumn{2}{|c|}{ Días/Days } & \multicolumn{2}{|c|}{ Días/Days } \\
\hline & 7 & 14 & 21 & 28 & 90 & 28 & 90 & 28 & 90 \\
\hline $1: 1,5: 3: 1,3$ & 40,00 & 71,00 & 83,00 & 88,00 & 84,00 & 0,65 & 0,75 & 0,43 & 0,60 \\
\hline $1: 1,5: 2: 1,3$ & 31,00 & 60,00 & 73,00 & 79,00 & 82,00 & 0,45 & 0,55 & 0,35 & 0,45 \\
\hline $1: 1,5:-: 1,2$ & 25,00 & 49,00 & 65,00 & 72,00 & 76,00 & 0,60 & - & 0,32 & 0,41 \\
\hline $1: 1: 3: 1,1$ & 43,00 & 72,00 & 69,00 & 67,00 & 67,00 & - & - & 0,37 & 0,37 \\
\hline $1: 1: 2: 1,1$ & 30,00 & 68,00 & 79,00 & 83,00 & 89,00 & 0,55 & 0,80 & 0,44 & 0,53 \\
\hline $1: 1:-.1,1$ & 15,00 & 33,00 & 53,00 & 65,00 & 70,00 & 0,55 & 0,50 & 0,31 & 0,44 \\
\hline $1: 0,5: 3: 1$ & 52,00 & 77,00 & 75,00 & 73,00 & 76,00 & - & - & 0,44 & 0,56 \\
\hline $1: 0,5: 2: 1$ & 23,00 & 66,00 & 79,00 & 81,00 & 83,00 & 0,45 & 0,65 & 0,40 & 0,53 \\
\hline $1: 0,5:-: 0,9$ & 22,00 & 36,00 & 51,00 & 68,00 & 80,00 & 0,45 & 0,65 & 0,41 & 0,43 \\
\hline
\end{tabular}

\section{TABLA III/TABLE III}

Granulometría fina, $1.000^{\circ} \mathrm{C}$. Conservadas al aire

(Fine granulometry, $1.000^{\circ} \mathrm{C}$. Air kept test pieces)

\begin{tabular}{|c|c|c|c|c|c|c|c|c|c|c|c|}
\hline \multirow[t]{3}{*}{$\begin{array}{c}\text { A m as ad as } \\
\text { Mixings }\end{array}$} & \multicolumn{6}{|c|}{$\begin{array}{l}\text { Durezas superficiales Shore C } \\
\text { Shore C superfitial hardness }\end{array}$} & \multicolumn{2}{|c|}{$\begin{array}{c}\text { Resistencia a flexión } \\
\text { en MpalFlexion } \\
\text { strength Mpa }\end{array}$} & \multicolumn{3}{|c|}{$\begin{array}{c}\text { Resistencia a } \\
\text { compresión en M pa } \\
\text { Compressive strength } \\
M p a\end{array}$} \\
\hline & \multicolumn{6}{|c|}{ Días/Days } & \multicolumn{2}{|c|}{ Días/Days } & \multicolumn{3}{|c|}{ Días/Days } \\
\hline & 7 & 14 & 21 & 28 & 90 & 120 & 28 & 90 & 28 & 90 & 120 \\
\hline $1: 1,5: 2: 1$ & 80 & 94 & 94 & 94 & 94 & 94 & 05 & - & 3,23 & 4,20 & * \\
\hline $1: 1,5: 2: 1,2$ & 60 & 90 & 94 & 93 & 93 & 99 & 1,75 & 1,55 & 1,85 & 2,50 & * \\
\hline $1: 1,5: 2: 1,1$ & 72 & 93 & 93 & 94 & 95 & 93 & 1,85 & 1,75 & 2,50 & 3,90 & * \\
\hline $1: 1,5: 2: 1,3$ & 55 & 87 & 91 & 92 & 94 & 91 & 1,00 & 1,20 & 1,50 & 2,20 & 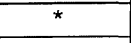 \\
\hline $1: 1,5:-: 1,1$ & 44 & 74 & 88 & 88 & 89 & 91 & 0,95 & 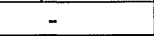 & 2,50 & 3,40 & 3,70 \\
\hline $1: 1,5:-: 1$ & 55 & 80 & 92 & 93 & 93 & 93 & 0,95 & 1,55 & 3,50 & 4,00 & 4,75 \\
\hline $1: 1,5:-: 1$ & 54 & 80 & 92 & 92 & 9 & 94 & 1,20 & 1,50 & 3,30 & 4,00 & 4,40 \\
\hline $1: 1,5:-: 1$ & 55 & 81 & 91 & 92 & 93 & 94 & 1,30 & 1,35 & 3,20 & 4,00 & 4,40 \\
\hline $1: 2:-: 1,2$ & 62 & 90 & 90 & 91 & 91 & 91 & 0,80 & 1,35 & 2,60 & 2,60 & 3,10 \\
\hline $1: 2,5:-: 1,4$ & 58 & 88 & 89 & 89 & 89 & 89 & - & 1,30 & 2,44 & 2,40 & 2,80 \\
\hline $1: 3:-: 1,5$ & 62 & & 89 & 89 & 89 & 89 & 0,75 & 0,55 & 2,66 & 2,90 & 3,10 \\
\hline
\end{tabular}




\section{TABLA IVITABLE IV}

Granulometría fina, $1.000^{\circ} \mathrm{C}$. Conservadas sumergidas

(Fine granulometry, $1.000^{\circ} \mathrm{C}$. Water immersed pieces)

\begin{tabular}{|c|c|c|c|c|c|c|c|c|c|c|c|}
\hline \multirow[t]{3}{*}{$\begin{array}{c}\text { A m as ad as } \\
\text { Mixings }\end{array}$} & \multicolumn{6}{|c|}{$\begin{array}{c}\text { Durezas superficiales Shore C } \\
\text { shore C superfitial hardness }\end{array}$} & \multicolumn{2}{|c|}{$\begin{array}{c}\text { Resistencia a flexión } \\
\text { en M palflexion } \\
\text { strength Mpa }\end{array}$} & \multicolumn{3}{|c|}{$\begin{array}{c}\text { Resistencia a } \\
\text { compresión en M pa } \\
\text { Compressive strength }\end{array}$} \\
\hline & \multicolumn{6}{|c|}{ Días/Days } & \multicolumn{2}{|c|}{ Días/Days } & \multicolumn{3}{|c|}{ Días/Days } \\
\hline & 7 & 14 & 21 & 28 & 90 & 120 & 28 & 90 & 28 & 90 & 120 \\
\hline $1: 1,5: 2: 1$ & 49 & 63 & 66 & 68 & 8 & 89 & 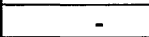 & 2,05 & 1,50 & 4,50 & * \\
\hline $1: 1,5: 2: 1,2$ & 21 & 38 & 47 & 48 & 78 & 83 & 0,65 & 2,35 & 0,70 & 2,50 & * \\
\hline $1: 1,5: 2: 1,1$ & 32 & 44 & 55 & 56 & 82 & 83 & 0,60 & 2,70 & 0,90 & 3,70 & * \\
\hline $1: 1,5: 2: 1,3$ & 16 & 31 & 43 & 45 & 74 & 81 & 0,50 & 1,90 & 0,60 & 1,90 & * \\
\hline $1: 1,5:-: 1,1$ & 23 & 38 & 39 & 44 & 72 & 74 & 0,75 & 2,35 & 0,90 & 3,30 & 3,75 \\
\hline $1: 1,5:-: 1$ & 31 & 44 & 48 & 51 & 77 & 80 & 1,10 & 3,10 & 1,20 & 4,50 & 4,80 \\
\hline $1: 1,5:-: 1$ & 34 & 44 & 49 & 51 & 76 & 81 & 0,80 & 2,15 & 1,25 & 4,40 & 4,40 \\
\hline $1: 1,5:-: 1$ & 36 & 45 & 50 & 52 & 78 & 82 & 1,00 & 2,90 & 1,50 & 4,90 & 5,25 \\
\hline $1: 2:-: 1,2$ & 28 & 41 & 47 & 51 & 78 & 83 & 0,80 & - & 1,10 & 4,00 & 5,25 \\
\hline $1: 2,5:-: 1,4$ & 34 & 50 & 55 & 57 & 79 & 82 & 1,20 & 2,35 & 1,20 & 3,90 & 4,90 \\
\hline $1: 3:-: 1,5$ & 38 & & 60 & 61 & 82 & 85 & - & 1.90 & 130 & 4,10 & 5,70 \\
\hline
\end{tabular}

* Conservadas para romper a mayoredad.

* Kept to be analyzed at later time.
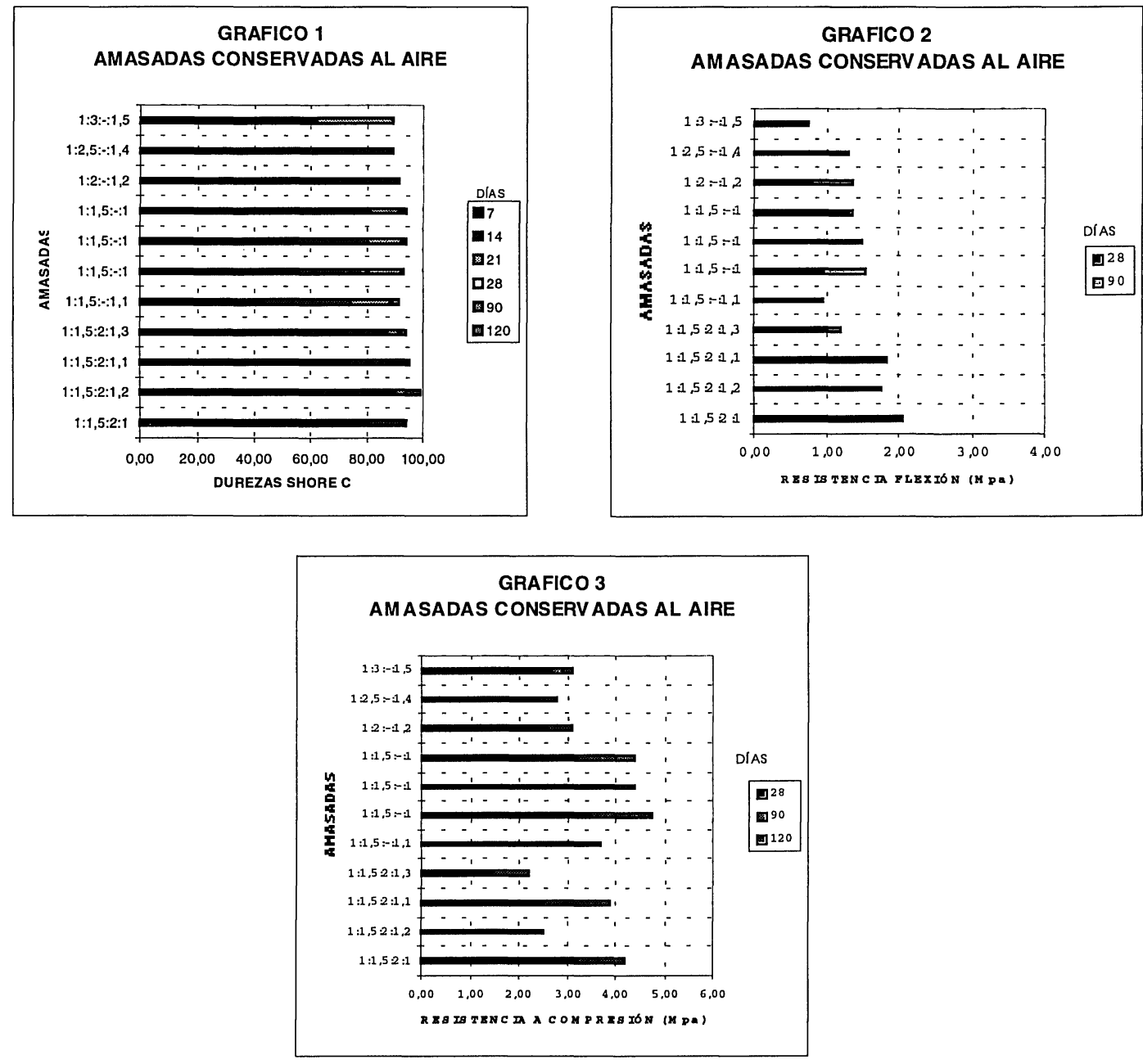

Gráficos 1, 2 y 3: muestras amasadas conservadas al aire. Graphics 1, 2 and 3: air kept mixings pieces. 

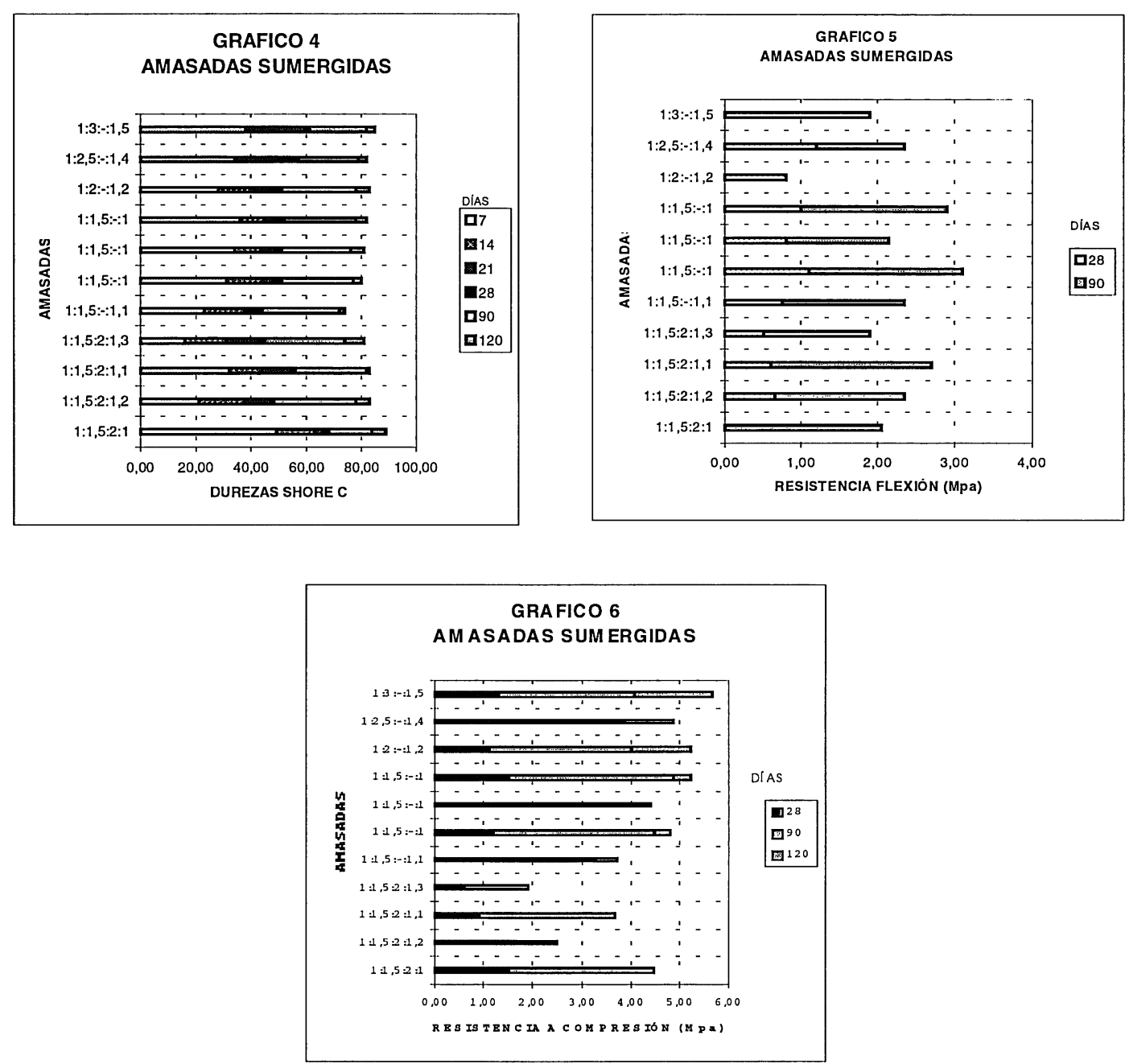

Gráficos 4, 5 y 6: muestras amasadas sumergidas. Graphics 4, 5 and 6: immersed mixings pieces.

de forma irregular, dándose los mayores aumentos a la edad de 90 días. El incremento de cantidad de agua en las amasadas no es tan determinante como en las probetas conservadas al aire, siempre que se aumente también la cantidad de chamota.

Para resistencias a flexión los mejores resultados se obtienen para proporciones de chamota de 1,5 sin arena o con arena, consiguiendo un incremento importante de resistencias a 90 días, superando incluso a las conservadas al aire.

Para resistencias a compresión sirven las mismas consideraciones que para resistencias a flexión, superando también a los valores obtenidos en las amasadas conservadas al aire. the greater increases appear at 90 days. The water quantity increase in the mixings is not determinant in the air kept test pieces, as long as there is an increase in chamotte quantity.

For flexion strength, better results are obtained with chamotte proportions 1.5, with or without sand, obtaining a meaningful increase of strength at 90 days, overpassing those of the air kept test pieces.

For compressive srength the same considerations can be made as the ones expressed for flexion strength, overpassing values of mixings kept in the air. 
3.4. Resultados del análisis microestructural obtenidos con chamotas cocidas a $1.000^{\circ} \mathrm{C}$ y granulometría fina, módulo granulométrico 0,32 .

Los resultados del análisis microestructural realizado han sido:

\section{. Estereomicroscopía}

Las muestras conscrvadas al aire tienen, en ambos casos, un aspecto más blanquecino.

Las muestras conservadas sumergidas en agua presentan un aspecto más reaccionado.

\section{- Microscopía electrónica}

En todos los casos se apreció un aspecto más denso de las muestras sumergidas.

No se aprecian, sin embargo, diferencias notables entre las dos dosificaciones analizadas para el mismo ticmpo y condiciones de conservación.

En la Foto 1 puede observarse el detalle de microestructura de la amasada 1:1,5:-:1 conservada al aire a la edad de 120 días y en la Foto 2, la misma amasada, pero conservada sumergida en agua.

En la Tabla V se presentan los resultados del microanálisis realizado por energías dispersivas en zonas puntuales de las diferentes muestras.

En dicha tabla se aprecia lo siguiente:

Hay una mayor relación $\mathrm{SiO}_{2} / \mathrm{CaO}$ en las muestras sumergidas en agua.

Las muestras conservadas sumergidas en agua tienen además más aluminio, potasio y hierro.

En general, se observa menos calcio y sodio en las muestras en agua.

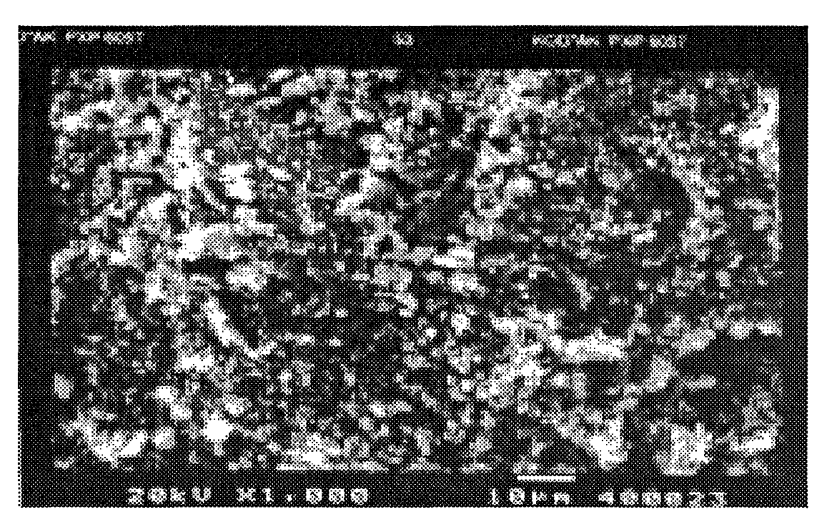

Foto $1 /$ Photo 1
3.4. Results obtained by the micro structural analysis with chamotte burned at $1.000^{\circ} \mathrm{C}$ and $f$ ine granulometry, granulmetric module 0.32 .

The results of the microstructural analysis performed have been:

\section{. Stereomicroscopy}

The air kept test pieces have a more whitish appearance.

The water immersed test pieces present greater reactionary aspect.

\section{. Electronic microscopy}

In all cases, denser aspect was shown on water immersed test pieces.

But there are notable differences to be signaled between the proportions analyzed for the same period of time and conservation conditions.

In Photo number 1 the detail of microstructure mixing 1:1,5-:1 kept at room temperature and at 120 days can be seen. In Photo number 2, the same mixing but kept immersed in water:

On table $V$ several results of microanalysis performed by dispersing energies at singular points in different test pieces are shown.

On the table there is:

A greater relation $\mathrm{SiO}_{2} / \mathrm{CaO}$ in the water immersed test pieces.

Those test pieces show also more aluminium, potassium and iron.

In general terms, there is less calcium and sodium in the water immersed test pieces.

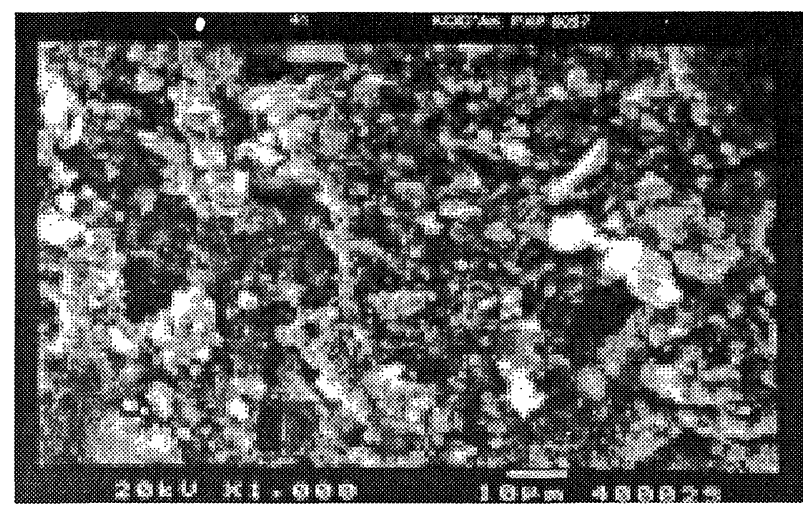

Foto 2 /Photo 2 
TABLA V/TABLE $V$

Resultados del análisis microestructural Microstructural analysis results

\begin{tabular}{|c|c|c|c|c|}
\hline \multirow{3}{*}{$\begin{array}{l}\text { Amasadas } \\
\text { Mixings }\end{array}$} & \multicolumn{4}{|c|}{ PROPORCIÓN DE COMPONENTESICOMPONENT PROPORTION } \\
\hline & \multicolumn{2}{|c|}{ 1:1,5:-:1 } & \multicolumn{2}{|c|}{ 1:2:-:1,2 } \\
\hline & Al aire/Air kept & $\begin{array}{c}\text { Sumergida } \\
\text { Water } \\
\text { immersed }\end{array}$ & Al aire/Air kept & $\begin{array}{c}\text { Sumergida } \\
\text { Water } \\
\text { immersed }\end{array}$ \\
\hline $\mathrm{Na}_{\mathbf{2}} \mathrm{O}$ & 0,99 & 0,50 & 1,35 & 0,62 \\
\hline MgO & 2,04 & 2,20 & 2,64 & 2,13 \\
\hline $\mathrm{Al}_{2} \mathrm{O}_{3}$ & 6,99 & 8,41 & 8,35 & 9,12 \\
\hline $\mathrm{SiO}_{2}$ & 24,03 & 26,52 & 26,87 & 29,80 \\
\hline $\mathrm{SO}_{3}$ & 1,69 & 0,63 & 1,30 & 1,65 \\
\hline $\mathrm{K}_{2} \mathrm{O}$ & 2,43 & 3,21 & 2,67 & 3,01 \\
\hline $\mathrm{CaO}$ & 58,75 & 54,15 & 52,42 & 48,79 \\
\hline $\mathrm{Fe}_{2} \mathrm{O}_{3}$ & 3,08 & 4,37 & 4,40 & 4,89 \\
\hline $\mathrm{SiO}_{2} / \mathrm{CaO}$ & 0,41 & 0,49 & 0,51 & 0,61 \\
\hline $\mathrm{Al}_{2} \mathrm{O}_{3} / \mathrm{Fe}_{2} \mathrm{O}_{3}$ & 0,44 & 0,52 & 0,53 & 0,54 \\
\hline
\end{tabular}

3.5. Resultados del análisis de difracción por $\mathrm{RX}$ obtenidos con chamotas cocidas a $1.000^{\circ} \mathrm{C}$ y granulometría fina, módulo granulométrico 0,32.
3.5. Diffraction by $R X$ analysis results obtained with chamotte burned at $1.000{ }^{\circ} \mathrm{C}$ and fine granulometry, granulemetric module 0.32 .

TABLA VI/TABLE VI

Identificación de DRX

DRX identification

\begin{tabular}{|c|c|c|c|c|c|c|}
\hline \multirow{2}{*}{$\begin{array}{l}\text { Fases } \\
\text { Identiflcad } \\
\text { as } \\
\text { lolentified } \\
\text { phases }\end{array}$} & \multicolumn{3}{|c|}{ Al alre/Air kept } & \multicolumn{3}{|c|}{ Sumergldas/Water Immersed } \\
\hline & $\begin{array}{l}7 \text { dias } \\
7 \text { days }\end{array}$ & $\begin{array}{l}28 \text { dias } \\
28 \text { days }\end{array}$ & $\begin{array}{l}120 \text { dias } \\
120 \text { days }\end{array}$ & $\begin{array}{l}7 \text { dias } \\
7 \text { days }\end{array}$ & $\begin{array}{l}28 \text { dias } \\
28 \text { days }\end{array}$ & $\begin{array}{l}120 \text { dias } \\
120 \text { days }\end{array}$ \\
\hline CH & +++ & +++ & ++ & +++ & ++ & + \\
\hline$\hat{C C}$ & + & ++ & +++ & + & + & + \\
\hline $\mathbf{S}$ & +++ & +++ & +++ & +++ & ++ & + \\
\hline SA & $\circ$ & 0 & 0 & 0 & - & - \\
\hline SAC & + & + & + & ++ & +++ & ++ \\
\hline SACH & 0 & $\circ$ & 0 & + & ++ & +++ \\
\hline
\end{tabular}

\section{LISTA DE ABREVIATURAS}

CH; - Hidróxido de calcio, C; - Carbonato de calcio, S; - Cuarzo, SA; - Silicato alumínico, SAC; - Silico aluminato de calcio, SACH; - Silico aluminato de calcio hidratado

\section{LISTA DE SÍMBOLOS}

$$
\begin{aligned}
& ++: \text { abundante } \\
& ++: \text { moderado } \\
& +: \text { poco } \\
& \text { o: trazos } \\
& +: \text { ausente }
\end{aligned}
$$

\section{CODE LIST}

CH-Calcium hydroxide, $\mathrm{C}$ - Calcium carbonate, $S$ - quartz, SAAluminum silicate, SAC-Calcium aluminate silicate, SACHHydrated aalcium aluminate silicate

\section{SYMBOL LIST}

+H: abundant

++ moderate

t: few

o: barely

-: absent 
Como puede obscrvarse en la Tabla VI, en las probetas conservadas al aire se van formando,

fundamentalmente, compuestos de carbonato de calcio a mayor cantidad en función de la edad, formándose también silico aluminato de calcio hidratado.

En las probetas que se han mantenido sumergidas en agua predominan los silico aluminatos de calcio hidratados o sin hidratar.

Estos resultados confirman los anteriormente obtenidos en el análisis microestructural y concuerdan con los de durezas Shore $\mathrm{C}$ y resistencias mecánicas a flexión y compresión, dando a largo plazo mejores resultados las probetas sumergidas en agua por la formación de compuestos tipo cementicio.

\section{CONCLUSIONES}

A partir de un análisis teórico fundamentado en el comportamiento de la cal y de sus morteros, con adición de chamota y arena, se han realizado una serie ensayos con mezclas de cal aérea apagada, chamota, arena y agua, mediante los que se ha determinado de modo experimental su comportamiento físico y mecánico, así como su composición por difracción por rayos $\mathrm{X}$ y análisis microestructural. Del análisis e interpretación de los resultados, se pueden obtener las siguientes conclusiones:

1. Se pueden modificar y mejorar las características mecánicas y físicas de los morteros de cal mediante la adición de chamota ó polvo de ladrillo.

2. Para chamotas cocidas a $1.300{ }^{\circ} \mathrm{C}$, correspondientes a procesos de gresificación, con independencia de la granulometría, además de no inferir propiedades hidráulicas al mortero, las resistencias mecánicas de aquellos no son válidas para la elaboración de morteros de agarre de fábricas de ladrillo.

3. Para temperaturas de cocción de la chamota de $1.000^{\circ} \mathrm{C}$, correspondiente a residuos de ladrillos cerámicos y tejería, en términos generales, a menor tamaño en la granulometría empleada se obtienen mejores resultados, en cuanto a resistencias mecánicas. La granulometría fina en este tipo de chamota confiere propiedades hidráulicas al mortero. Sin embargo, la granulometría gruesa no se las conficre.

4. La arena, en general, y para el uso de mortero de agarre para fábricas de ladrillo, no mejora las condiciones del mortero. Por los resultados obtenidos se puede utilizar la arena en pequeñas proporciones, del orden de $1: 1$ en relación en peso con respecto a la cal.
As can be seen on table VI, in the air kept test pieces, more calcium carbonate compounds are formed according to the age. Also, hydrated calcium aluminate silicate is produced.In the water immersed test pieces there is a predominance of calcium aluminate silicate both hydrated and non-hydrated.

These results confirm the previously oblained results for the micro structural analysis and are in agreement with the Shore $C$ hardness, and the flexion and compressive strengths. The water immersed test pieces show better results in the long run due to the formation of cement type compounds.

\section{CONCLUSIONS}

From a theoretical analysis based on the lime and mortars behaviors, with the addition of sand and chamotte, several tests have been performed with mixings of aired lime, chamotte, sand and water. The mechanical and physical behavior has been experimented as well as its composition by $X$ ray and microstructural analysis. The following conclusions can be draw'n from the analysis and results obtained:

\section{Mechanical and physical characteristics of lime mortars can be improved by the addition of chamotte or brick powder.}

2. For chamottes burned at $1.300{ }^{\circ} \mathrm{C}$, corresponding to earthenware processes, and leaving aside the granulometry, the mechanical strengths are not valid for the manufacture of keying mortars for brickwork.

3. For burning temperatures of chamotte of $1.000^{\circ} \mathrm{C}$, corresponding to ceramic brick and tile waste, the smaller the granulometry size, the better the result obtained when looking at mechanical strengths. Fine granulometry in this type of chamotte gives hydraulic properties to the mortars, but not so the course granulometry.

\section{Sand, in general, does not improve mortar} conditions in brickwork mortars. Small amounts of sand can be used, such as 1:1 lime/weight proportion. 
5. A edades tempranas, habitualmente, se aprecia un mejor comportamiento físico y mecánico de las probetas conservadas al aire. Sin embargo, a mayor edad se van igualando los resultados con respecto a las probetas conservadas sumergidas en agua, llegando éstas a superar incluso a las conservadas al aire en valores de resistencias.

6. La mejor dosificación para morteros de agarre de fábricas de ladrillo, tanto en restauración como en obra nueva, resulta ser la que contiene una relación en peso, con respecto a la cal, comprendida entre 1,5 y 2 de chamota (con granulometría fina y temperatura de cocción entorno a $\operatorname{los} 1.000^{\circ} \mathrm{C}$ ), y entre 1 y 1,5 de agua. Asimismo, tal y como ha quedado expuesto en el punto cuarto de estas conclusiones, se puede añadir arena en relación de 1 en peso con respecto a la cal.

7. Los análisis microestructurales confirman la aparición de compuestos hidráulicos en los morteros de cal y chamota, así realizados

\section{AGRADECIMIENTOS}

Los autores del presente trabajo desean expresar su agradecimiento al Dr. D. Demetrio Gaspar Tébar, al Dr. D. José Luis Sagrera Moreno y a Dña. Esperanza Menéndez Méndez por la ayuda prestada.
5. At an early age. a better physical and mechanical behavior can be observed in air kept test pieces. Nevertheless, later in time the results are balanced in relation to the water immersed test pieces. The latter even overpass the strength values of air kept test pieces.

6. The best proportion for keying brickwork mortars, in restoration works as well as in new constructions, is the one with a weight/lime relationship between 1.5 and 2 and of chamotte (with fine granulometry and burning temperature of about $\left.1.000^{\circ} \mathrm{C}\right)$, and water in a proportion of 1 and 1.5 .

\section{The micro structural analysis confirm the appearance of hydraulic compounds in lime and} chamotle mortars.

\section{ACKNOWLEDGEMENTS}

The authors of this article wish to thank PhD. Demetrio Gaspar Tébar, PhD..José Luis Sagreda Moreno and Ms. Esperanza Menénde: Méndes for their great help.

\section{BIBLIOGRAFÍA}

(1) G. Baronio, L. Binda and N. Lombardini: The role of brick pebbles and dust in conglomerates based en hydrated lime and crucrushed brichs Constructions and building materials. vol. II. n"1, pp 33-40. Elsevier science Itd. 1997.

(2) G.T. Suter, J. Vandenberg and L. Fontaine: Bond capacity of mortars for historic structures. The journal of preservation technology. A.P.T. Bulletin. Vol. XXVII, n" 4, pp 27-35. 1996.

(3) González Cortina, Mariano y Villanueva Domínguez, Luis de . Ensayo físico de hidraulicidad. V Jornada Nacional. Aplicaciones arquitectónicas de materiales. Abril 1999

(4) E. Liebig, E. Althaus: Kaolinit und Montmorillonit als puzzolanische komponenten in kalkmörteln-unbehandelt und nach termischer aktivierung. ZKG International. pp282-290. 1997.

(5) G. Baronio and L. Binda: Study of the pozzolanicity of some bricks and clays. Constructions and building materials. vol. II, $\mathrm{n}^{\circ} 1$, pp 41 -46. Elsevier science ltd. 1997.

NOTA: El presente trabajo se ha desarrollado en el marco del proyecto de investigación MAT 1997-1031, “ Efectos sinérgicos en materiales compuestos de matriz conglomerada y adiciones de bajo costo ", subvencionado por la Subdirección General de Proyectos de Investigación del Ministerio de Ciencia y Tecnología.

NOTE: This research has been accomplished within the MAT 1997-1031 investigation project: "Synergic effects on compound materials of conglomerated matrix and low cost addition" supported by the Subdirección General de Proyectos de Investigación from the Ministerio de Ciencia y Tecnologia. 